\title{
COULD THE TOTAL SPINE MRI BE USEFUL FOR DECISION-MAKING IN PATIENTS WITH ANKYLOSIS SPONDYLITIS? DATA FROM A 2-YEAR PROSPECTIVE STUDY
}

Louise Tandaya Bendahan ${ }^{1, \star}$, Thauana Luiza de Oliveira ${ }^{1}$, Eloy de Avila Fernandes ${ }^{1}$, Artur da Rocha Correa Fernandes ${ }^{1}$, Carla Goncalves Schahin Saad ${ }^{1}$, Marcelo de Medeiros Pinheiro ${ }^{1}$

1.Universidade Federal de São Paulo, São Paulo (SP), Brazil.

*Corresponding author: louisebendahan@globo.com

\section{BACKGROUND}

The lack of specific biomarkers to evaluate disease activity in patients with ankylosing spondylitis (AS) is a relevant clinical challenge. The aim was to assess the role of total spine magnetic resonance imaging (MRI) in AS patients with clinical doubt between activity or chronicity.

\section{METHODS}

A total of 57 AS patients, according to the New York criteria, were included in this 2-year prospective study. The inclusion criterion was the clinical doubt between to be or not to be with disease activity, considering standard tools, including inflammatory back pain, peripheral involvement, extra-articular manifestations and C-reactive protein (CRP). The patients were divided in two groups (A: pain + low CRP; B: no symptoms + high CRP). All patients performed total spine MRI and the reading was performed by blinded radiologist, considering SPARCC methodology. After that, an expert rheumatologist used it to make major clinical decision. A positive spine MRI was defined when three or more bone marrow edema signals were found, according to the OMERACT.

\section{RESULTS}

Positive MRI was observed in 40 and $30 \%$ of group A and B, respectively. Using the global evaluation (clinical, lab and imaging information) performed by expert rheumatologist as gold-standard, the concordance between positive MRI and overall disease activity was observed in almost $70 \%$ of AS patients $(r=0.36, p=0.01)$, especially in group $A$. On the other hand, higher inflammation severity and area score was more found in group $B(15.7 \pm 7.5$ and $9.9 \pm 6.7 ; p=0.048$, respectively). After 12 and 24 month follow-up, most of the patients remained with the same medical decision, suggesting good assertiveness over time.

\section{CONCLUSION}

Our data showed whole spine MRI could be used as an important tool for a suitable clinical decision between disease activity and other non-inflammatory causes of pain in patients with AS.

\section{KEYWORDS}

Ankylosing spondylitis, Disease activity, Magnetic resonance imaging, Clinical management, Making decision. 